\title{
Modeling of Creep Effect in DMSP-5 Fluidic Muscle
}

\author{
Monika Trojanová, Alexander Hošovský, Tomáš Čakurda \\ Technical University of Košice, Faculty of Manufacturing, Technologies with a seat in Prešov, \\ Bayerova 1, Prešov, Slovakia
}

\begin{abstract}
The presence of effects as hysteresis or creep effect may adversely affect the control performance of a fluid muscle-driven manipulator. Due to this fact, the authors of the article research the influence and properties of phenomena with slow dynamics of fluid muscles on the accuracy of their modeling. To investigate these phenomena, the authors designed and constructed an experimental apparatus that allows the investigation of the dynamic characteristics of a particular fluid muscle from the manufacturer FESTO.
\end{abstract}

Keywords - Creep Effect, Dynamics, Fluid Muscle, Modeling.

\section{Introduction}

The manipulator applied in the industry has to meet the conditions of flexibility and safety in cooperation with humans. In that case, such devices' many times standard drives are not sufficient to achieve the required level of defined properties. This involves the application of unconventional types of actuators, respectively soft actuators [1], [2], [3].

One such unconventional kind of actuator is, for example, fluid muscles from FESTO [4], [5], which

DOI: $10.18421 /$ TEM111-03

https://doi.org/10.18421/TEM111-03

Corresponding author: Monika Trojanová, Technical University of Košice, Faculty of Manufacturing, Technologies with a seat in Prešov, Prešov, Slovakia.

Email: monika.trojanova@tuke.sk

Received: 07 November 2021.

Revised: 18 December 2021.

Accepted: 27 December 2021.

Published: 28 February 2022.

(c) BY-NC-ND(C) 2022. Monika Trojanová, Alexander Hošovský \& Tomáš Čakurda; published by UIKTEN. This work is licensed under the Creative Commons AttributionNonCommercial-NoDerivs 4.0 License.

The article is published with Open Access at https://www.temjournal.com/ were developed from pneumatic artificial muscle of the McKibben type [6], [7].

When applying actuators in practice, it is necessary to consider all positive and negative aspects.

The following positives characterize FESTO muscles:

- high level of safety in human-machine interaction;

- use in more demanding operating conditions (ambient temperature up to $60^{\circ} \mathrm{C}$, dust, humidity);

- high ratio of produced tensile force to weight and others.

The muscles produce the traction force due to a change in the pressure of the working medium inside the muscle, which leads to a contraction (change in the length of the muscle). Negative, respectively undesirable properties of fluid muscles that affect the accuracy of control are:

- nonlinear characteristics related to the design of the muscle - the use of elastic materials;

- presence of hysteresis;

- presence of creep effect and others.

The above-mentioned undesirable properties can significantly affect the modeling and control of this actuator and therefore need to be analyzed. There is considerable interest in researching the abovementioned unwanted properties, mainly due to this type of actuator's benefits. The latest research results in modeling the hysteresis of pneumatic artificial muscles are summarized, e.g., in publications [8], [9], [10]. In [8], the authors propose a modified asymmetric generalized Prandtl-Ishlinkii model for the asymmetric irregular hysteresis of FESTO muscle. On the contrary, in [10], the feedforwardfeedback composite control method is proposed, intended to compensate the hysteresis of pneumatic artificial muscles involved in the delta mechanism. The study of fluid muscle hysteresis often overlaps with the study of the creep effect, as these properties interact with each other. The authors of the article [11] describe the modeling of hysteresis using the Maxwell-slip model and examine the properties of 
the creep effect. The creep effect is a dynamic phenomenon in which the displacement of a muscle changes slowly over time due to the elasticity of the muscle material. From the published articles [11], [12], [13], it is known about the creep effect that the temperature of the environment in which the muscle is located, and the static pressure affect the creep effect; dependence of the future development of the creep effect on the previous one; stabilization of this phenomenon after a certain time and others. Although several researchers have studied the creep effect in fluid muscles, this area is still less studied than hysteresis.

Due to this fact, the authors of the article created an experimental apparatus at their workplace, which will allow the measurement and investigation of the characteristics associated with the creep effect of one fluid muscle. In addition to the introduction and conclusion, the article contains chapters devoted to the description of the experimental apparatus; a description of the measured data, and then a section summarizing the results of research into the creep effect on muscle displacement, specifically the identification of a dynamic mathematical model of muscle displacement at different muscle pressures.

\section{Experimental Apparatus}

Figure 1 shows a diagram of an experimental apparatus designed and assembled at the workplace of the author's team to investigate the influence and properties of phenomena with slow dynamics of fluid muscles on the accuracy of their modeling. The diagram interprets the most basic elements and components. The supporting structure consists of basic elements: $\mathbf{2}$ - Main frame, 3 - Temperature chamber, 4 - L-shaped profile, 8 - Mounting plate, and 9 - Transverse profile. Another group of components consists of electrical elements and elements enabling signal transmission and processing. These include 1 - Proportional pressure regulator, 7 Optical displacement sensor, 10 - Heating element, 11 - Temperature sensor, 12 - Digital thermostat, 13 Power supply, 14 - Input/output device, and 15 Computer. The last two elements are the object of research 5 - Fluid muscle and $\mathbf{6}$ - Load.

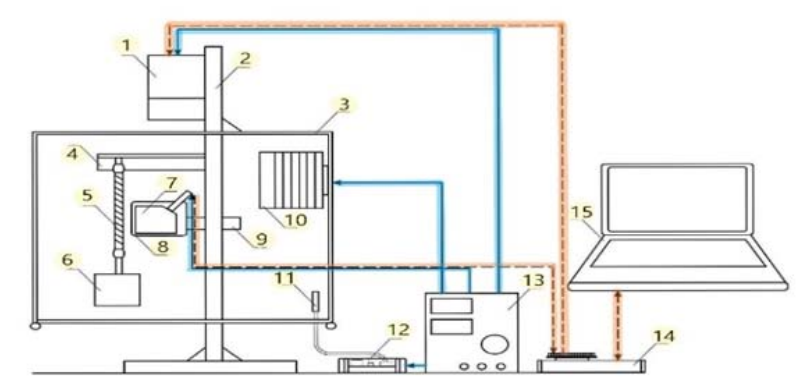

Figure 1. Scheme of the apparatus
One of the ends of the fluid muscle from FESTO is attached to the L-shaped profile using a screw connection, and the profile subsequently to the vertical part of the main frame structure. Since the apparatus will be used for isotonic measurements, constant weight on the muscle is ensured by a load attached to the muscle's other (free) end and a screw connection. Another condition for isotonic measurement is a change in muscle pressure, while pressure regulation is provided by a proportional pressure regulator, which is attached to the main frame. The source of the analog signal, which is used to control the pressure regulator, is a computer, which ensures the transmission and processing of the control signal via the input/output device. On the scheme, this signal is marked with a red dashed line. By applying a signal to the pressure regulator, the desired pressure in the muscle is ensured, to which compressed air from the compressor is also supplied (or the muscle is discharged in case of pressure reduction). One of the monitored variables in the isotonic measurement may be the displacement of the muscle, which is caused by the muscle contraction when the pressure in the muscle changes. An optical displacement sensor is built into the displacement sensor, which is mounted on the transverse profile. The measured displacement is in the form of a signal transmitted to the input/output device and then processed in the interpretation in the computer. This signal is also marked with a red dashed line in the scheme. The experimental apparatus was made to examine the creep effect of fluid muscles depending on the ambient temperature near the muscle. The muscle and some components are enclosed in a temperature chamber. The chamber's role is to provide thermal insulation, and subsequently, thermal regulation inside the chamber, and thus around the muscle using heating elements. These have the task of heating the chamber to the desired temperature while a temperature sensor senses the temperature. The sensor is connected to a digital thermostat, which switches the above heating elements. The chamber is made of plexiglass, which is transparent. At the same time, the two walls of the chamber are openable, which ensures access and easy handling inside the chamber with individual components. The power supply of electrical elements such as pressure regulator, heating elements, displacement sensor, and the digital thermostat is provided by the power supply (in the scheme, a continuous blue line marks this power supply in the direction from the power supply to the individual elements).

For the research described in this article, the experimental apparatus was adapted to measure the creep effect under isotonic conditions: 
- one end of the muscle firmly attached;

- at the other free end of the load with constant weight;

- input parameter muscle pressure;

- output monitored parameter of muscle displacement.

The fluid muscle FESTO DMSP 5 was used in the experiments. The overview of the basic parameters is given in Table 1. It is a type of muscle that has pressed ends intended for screw connection with integrated adapters.

Table 1. Parameters of Fluid muscle FESTO DMSP-5 [4]

\begin{tabular}{||lc||}
\hline \multicolumn{1}{|c|}{ Parameter } & Value \\
Diameter & $0.005 \mathrm{~m}$ \\
Length & $0.15 \mathrm{~m}$ \\
Theoretical force & $140 \mathrm{~N}$ \\
Operating pressure & $600 \mathrm{kPa}$ \\
Ambient temperature & $-5 \ldots+60^{\circ} \mathrm{C}$ \\
Max. permissible contraction & $20 \%$ of nominal \\
& length \\
\hline
\end{tabular}

\section{Measured Data from the Experimental System}

Isotonic measurements were performed to investigate the creep effect of FESTO muscle of the DMSP-5 type on the above-mentioned experimental apparatus with a constant muscle load $\mathrm{m}=1.2 \mathrm{~kg}$. The measurements were performed as follows:

- A simulation scheme was created in the Simulink, which allowed the input of voltage values in the range from $0.5 \mathrm{~V}$ to $5.5 \mathrm{~V}$ (which subsequently corresponded to muscle pressures from $30 \mathrm{kPa}$ to $330 \mathrm{kPa}$ ).

- A step signal was used to measure data set No. 1, where after five seconds, the value of muscle pressure changed to $30 \mathrm{kPa}$. The measurement lasted 100 seconds and was performed a total of 30 times under the same conditions.

- The monitored output parameter was muscle displacement, which was detected by the sensor, and the values were stored and processed in MATLAB and Simulink.

- A total of 11 data sets were obtained by measurements, varying depending on the pressure, which jumped to a value ranging from $30 \mathrm{kPa}$ to $330 \mathrm{kPa}$ in $30 \mathrm{kPa}$ increments over a measurement time of 5 th second.

- Each representative data set was subsequently adjusted so that the values from the 30 measurements were averaged to exclude possible measurement errors.

- The averaged output displacements were smoothed in the MATLAB environment using the SMOOTH command. In total, there were created 11-time dependences of the output muscle displacement depending on the step-change in pressure at 11 different pressures. The time courses of the averaged displacements for the individual pressures can be seen in Figure 2.

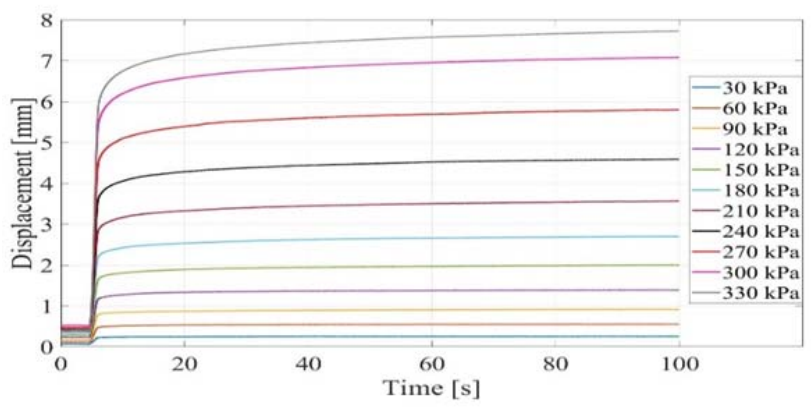

Figure 2. Time dependence of muscle displacement at different pressures

\section{Identification of the Models - results}

In the experimental part, an attempt was made to find such dynamic mathematical models for muscle displacement, which would also take into account the creep effect of the muscle.

\section{Nonlinear Creep Model}

The first model that was chosen to identify the measured data was the Nonlinear creep model. It takes into account the displacement in time and, at the same time, takes into account the creep rate of the actuator. In [14] in the third chapter, which is devoted to hysteresis modeling in smart actuators, the authors present an equation of such a model in the form:

$$
y_{c}(t)=y_{c 0}\left(1+\gamma_{c} \log \frac{t}{t_{0}}\right)
$$

where $y_{c}(t)$ is displacement in time $t, y_{c 0}$ is displacement in time $t_{0}$, and $\gamma_{c}$ is creep rate.

The Curve Fitting Toolbox, which is part of MATLAB, was used to find the nonlinear creep model of the measured displacement data. The input for this toolbox was X data - time matrix; the output was measured (averaged) displacement values for a specific pressure marked as Y data. Since the object of interest was, in particular, the time span from the fifth second, when the jump in the signal occurred, the displacement matrices were adjusted so that the first approximately 4500 samples were deleted from the original 100001 samples. The modified data were used to find the Nonlinear creep model.

In the toolbox, in the model type section, Custom Equation was selected, where Equation 1 was rewritten into the form:

$$
y=a\left(1+b \cdot \log \frac{x}{c}\right)
$$

where $x$ was the matrix of input data (time); $y$ was the matrix of output data (measured displacement for 
individual pressures); $a$ was the value of displacement in time 20th second; $c$ was time $t=20$ seconds; $b$ was creep rate.

The mathematical model was determined using the Levenberg-Marquardt algorithm.

The individual resulting models at different pressures were compared with the measured displacement values based on the statistical parameter Normal Root Mean Square Error (NRMSE) - (in this research named as Fit):

$$
\text { Fit }=\left(1-\frac{\sqrt{\sum_{j=1}^{n}\left[y_{j}-\bar{y}_{j}\right]^{2}}}{\sqrt{\sum_{j=1}^{n}\left[y_{j}-\frac{1}{n} \sum_{j=1}^{n} y_{j}\right]^{2}}}\right) \times 100 \%
$$

where $y_{j}$ system output on the k-th sample, $\bar{y}_{j}$ model output on the k-th sample, $n$ - number of samples. The value of the Fit indicator was given as a percentage. The output of the model corresponds to the desired output if the value Fit $=100 \%$, and thus the higher the Fit, the closer the model is to the expected state.

Table 2. Nonlinear creep models for individual pressures

\begin{tabular}{|ccc|}
\hline $\begin{array}{c}\text { Pressure } \\
{[\mathbf{k P a}]}\end{array}$ & Nonlinear creep model & $\begin{array}{c}\text { Fit } \\
{[\%]}\end{array}$ \\
30 & $\mathrm{y} 1=0.2402(1+0.04836(\log (\mathrm{t} / 20)))$ & 33.92 \\
60 & $\mathrm{y} 2=0.5280(1+0.04545(\log (\mathrm{t} / 20)))$ & 32.01 \\
90 & $\mathrm{y} 3=0.87(1+0.05017(\log (\mathrm{t} / 20)))$ & 34.62 \\
120 & $\mathrm{y} 4=1.3286(1+0.0506(\log (\mathrm{t} / 20)))$ & 32.91 \\
150 & $\mathrm{y} 5=1.8881(1+0.05763(\log (\mathrm{t} / 20)))$ & 36.43 \\
180 & $\mathrm{y} 6=2.529(1+0.06299(\log (\mathrm{t} / 20)))$ & 40.03 \\
210 & $\mathrm{y} 7=3.324(1+0.06496(\log (\mathrm{t} / 20)))$ & 42.01 \\
240 & $\mathrm{y} 8=4.281(1+0.06733(\log (\mathrm{t} / 20)))$ & 42.29 \\
270 & $\mathrm{y} 9=5.3888(1+0.0698(\log (\mathrm{t} / 20)))$ & 43.09 \\
300 & $\mathrm{y} 10=6.578(1+0.0709(\log (\mathrm{t} / 20)))$ & 43.13 \\
330 & $\mathrm{y} 11=7.1637(1+0.07211(\log (\mathrm{t} / 20)))$ & 42.43 \\
\hline
\end{tabular}

Table 2 provides an overview of the resulting nonlinear creep models as a function of pressure, which was identified in the Curve Fitting Toolbox with an indication of the achieved Fit parameter. The table shows that the Fit also increased with increasing pressure, but the identified models do not reach a sufficient level to be used later. The increasing tendency of the Fit parameter with increasing pressure is related to the fact that the higher the pressures in the muscles, the smoother the muscle displacements compared to the small pressures (which can also be seen from the measured courses of individual displacements).

In Figure 3, it is possible to see the time dependence of the resulting displacement of the best nonlinear creep model at a pressure of $300 \mathrm{kPa}$, which reached in comparison with the measured data Fit $=$ $43.13 \%$. In the graph, this output is shown by a red curve and marked in the legends as a model. The black curve represents the real measured output (desired state), and in the legend, it is marked as real data.

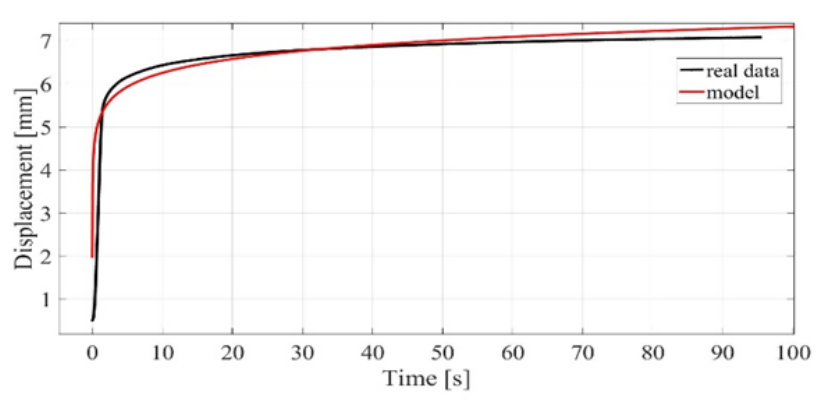

Figure 3. Nonlinear creep model for pressure $300 \mathrm{kPa}$

\section{Power Model}

Since no dynamic mathematical model was found within the nonlinear creep model that would reach the level of the Fit parameter at least $60 \%$, the Power model was chosen as the second type of model. The same toolbox as for the Nonlinear creep model was used to identify the dynamic model. The general form of the power model equation is expressed as follows:

$$
y=a \cdot x^{b}+c
$$

where:

- $\quad x$ was the matrix of input data (time);

- $y$ was the matrix of output data (measured displacement for individual pressures);

- $\quad a, b$ and $c$ are constants.

The inputs and outputs that were entered into the toolbox were the same as when creating the nonlinear creep model, and thus the input was the time and output measured displacement. The same algorithm was also used as in the first case - LevenbergMarquardt. In order to be able to compare the identified Power models with nonlinear creep models, the Fit parameter was also used for evaluation.

In Table 3, we can see an overview of the resulting power models, which were identified for pressures in the range of $30-330 \mathrm{kPa}$. As for the nonlinear creep model, the value of the Fit indicator increased with increasing pressure. However, the difference is in the values of the Fit parameter. While for nonlinear creep models, the Fit value ranged from $33.92 \%$ to $43.13 \%$, the Fit indicator for power models started from $51.68 \%$, and for the best model, it reached $60.76 \%$.

Table 3. Power models for individual pressures

\begin{tabular}{|ccc|}
\hline $\begin{array}{c}\text { Pressure } \\
{[\mathbf{k P a}]}\end{array}$ & Nonlinear creep model & $\begin{array}{c}\text { Fit } \\
{[\%]}\end{array}$ \\
30 & $\mathrm{Y} 1=-0.07645 \mathrm{t}^{-0.3166}+0.2733$ & 51.68 \\
60 & $\mathrm{Y} 2=-0.1819 \mathrm{t}^{-0.3164}+0.5983$ & 53.28 \\
90 & $\mathrm{Y} 3=-0.3264 \mathrm{t}^{-0.3066}+1.001$ & 53.99 \\
120 & $\mathrm{Y} 4=-0.5214 \mathrm{t}^{-0.3074}+1.533$ & 54.28 \\
150 & $\mathrm{Y} 5=-0.8336 \mathrm{t}^{-0.2938}+2.233$ & 55.64 \\
180 & $\mathrm{Y} 6=-1.176 \mathrm{t}^{-0.2877}+3.04$ & 57.68 \\
210 & $\mathrm{Y} 7=-1.605 \mathrm{t}^{-0.2819}+4.031$ & 59.73 \\
240 & $\mathrm{Y} 8=-2.175 \mathrm{t}^{-0.2779}+5.243$ & 60.02 \\
270 & $\mathrm{Y} 9=-2.838 \mathrm{t}^{-0.2749}+6.658$ & 60.56 \\
300 & $\mathrm{Y} 10=-3.551 \mathrm{t}^{-0.2732}+8.167$ & 60.76 \\
330 & $\mathrm{Y} 11=-3.946 \mathrm{t}^{-0.2745}+8.917$ & 60.21 \\
\hline
\end{tabular}




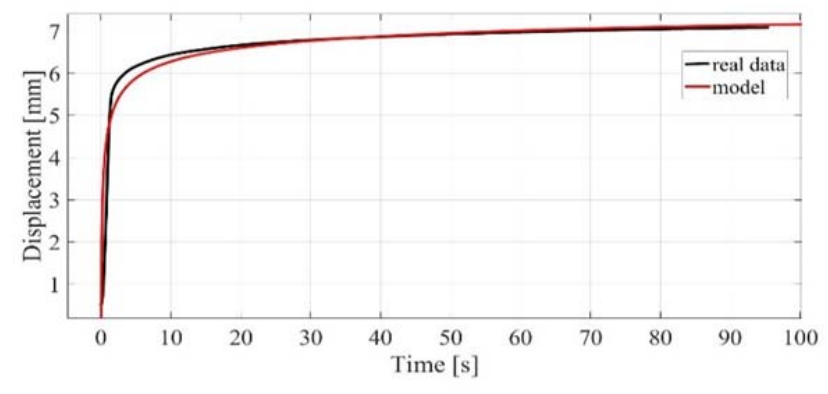

Figure 4. Power model for pressure $300 \mathrm{kPa}$

In Figure 4, it is possible to see the time dependence of the output of the best power model at a muscle pressure of $300 \mathrm{kPa}$ (red curve) when the Fit value reached $60.76 \%$ compared to the real measured data (black curve). Compared to the best nonlinear creep model, this power model can identify outputs from the initial values over time, while the creep model did not start until the offset value was around 2 $\mathrm{mm}$.

\section{Creep Rate}

Since the creep effect is a slowly occurring phenomenon over time, the creep rate was monitored within the measured values depending on the applied pressure (how many millimeters the muscle displacement per second changed at a steady constant speed at a specific muscle pressure). From the velocities, which were derived by derivatives of displacements (in MATLAB using the DIFF command), it was possible to observe that the stabilization of the velocity at each measurement after a step change in pressure occurred within ten seconds; therefore, the creep rate was chosen from 20 seconds to the end measurements (100th second).

In Figure 5, we can observe the dependence of the creep rate on the individual pressures used in the measurements. The dependence shows that with increasing pressure, the creep rate changes almost linearly and is therefore dependent on muscle pressure.

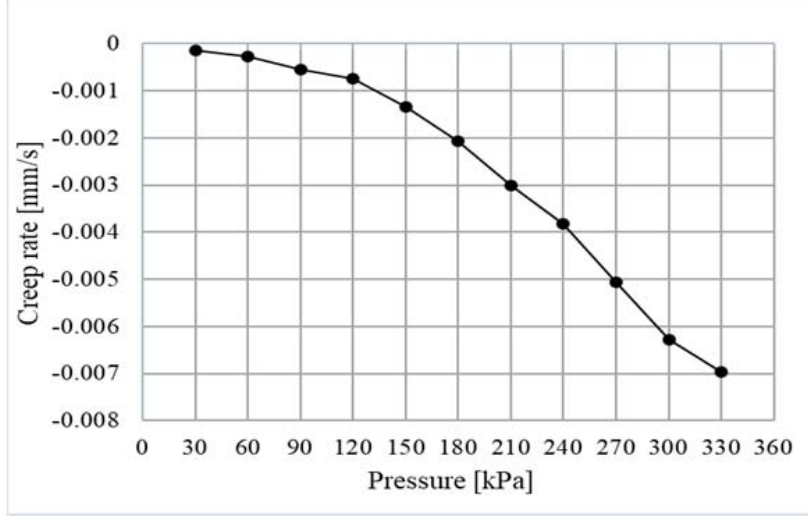

Figure 5. Creep rate depending on muscle pressure

\section{Conclusion}

The article addressed the issue of the influence of the creep effect on dynamic modeling of muscle displacement; respectively, the question of the dependence of the creep rate on the pressure in the fluid muscle was solved. Two types of dynamic mathematical models were compared. The first was the Nonlinear creep model, which defines muscle displacements over time, but also includes the creep rate. The second type was the Power model. Based on the Fit indicator (NRMSE parameter), the results of the individual models were compared, and it was shown that the Power model was more effective for the given muscle type and the assessed muscle pressures.

The dependence of the creep rate on the increasing muscle pressure was also demonstrated by measurements and subsequent analysis. In the next research, the authors would like to verify whether the creep rate dependence on increasing pressure also applies to larger range types of DMSP muscles, but also to what extent the Power model corresponds to measured data in the higher pressures and muscle displacements.

\section{Acknowledgements}

The research work is supported by Research grant for young researchers at TUKE, no. 05/TUKE/2021: "Research of the impact and the properties of phenomena with slow dynamics of fluid muscles on the accuracy of their modeling," and by grant VEGA 1/0393/18 "Research of Methods for Modeling and Compensation of Hysteresis in Pneumatic Artificial Muscles and PAM-actuated Mechanisms to Improve the Control Performance Using Computational Intelligence." 


\section{References}

[1]. Zhang, L., Qu, S., \& Du, X. (2021). Intelligent Soft Actuators and Flexible Devices. Advanced Intelligent Systems, 3(10).

[2]. Miriyev, A. (2019, December). A Focus on Soft Actuation. In Actuators (Vol. 8, No. 4, p. 74). Multidisciplinary Digital Publishing Institute.

[3]. Andrikopoulos, G., Nikolakopoulos, G., \& Manesis, S. (2011, June). A survey on applications of pneumatic artificial muscles. In 2011 19th Mediterranean Conference on Control \& Automation (MED) (pp. 1439-1446). IEEE.

[4]. FESTO. (2019). Fluidic Muscle DMSP/MAS. Retrieved from:

https://www.festo.com/rep/en_corp/assets/pdf/info_50 1 en.pdf [accessed: 19 September 2021].

[5]. Petre, I. M. (2021). An approach regarding some performances of a FESTO pneumatic muscle actuator. In IOP Conference Series: Materials Science and Engineering (Vol. 1009, No. 1, p. 012047). IOP Publishing.

[6]. Chou, C. P., \& Hannaford, B. (1994, May). Static and dynamic characteristics of McKibben pneumatic artificial muscles. In Proceedings of the 1994 IEEE international conference on robotics and automation (pp. 281-286). IEEE.

[7]. Tondu, B. (2012). Modelling of the McKibben artificial muscle: A review. Journal of Intelligent Material Systems and Structures, 23(3), 225-253.

[8]. Xie, S., Ren, G., \& Wang, B. (2020). A modified asymmetric generalized Prandtl-Ishlinskii model for characterizing the irregular asymmetric hysteresis of self-made pneumatic muscle actuators. Mechanism and Machine Theory, 149, 103836.
[9]. Luo, X., Xiao, M., Ding, Y., \& Ding, H. (2020). Hysteresis modeling and compensation of a pneumatic end-effector based on Gaussian process regression. Sensors and Actuators A: Physical, 315, 112227.

[10]. Geng, P., Qin, Y., Zhong, L., Qu, F., Bie, D., \& Han, J. (2020, October). Direct Inverse Hysteresis Compensation of a Pneumatic Artificial Muscles Actuated Delta Mechanism. In 2020 10th Institute of Electrical and Electronics Engineers International Conference on Cyber Technology in Automation, Control, and Intelligent Systems (CYBER) (pp. 1823). IEEE.

[11]. Minh, T. V., Kamers, B., Ramon, H., \& Van Brussel, H. (2012). Modeling and control of a pneumatic artificial muscle manipulator joint-Part I: Modeling of a pneumatic artificial muscle manipulator joint with accounting for creep effect. Mechatronics, 22(7), 923933.

[12]. Tri, V. M., Kamers, B., Ramon, H., \& Van Brussel, H. (2010). Characterization of Hysteresis in a Pneumatic Muscle Manipulator with Accounting for the Creep Effect. IFAC Proceedings Volumes, 43(21), 296-302.

[13]. Tri, V. M., Tjahjowidodo, T., Ramon, H., \& Van Brussel, H. (2011). A New Approach to Modeling Hysteresis in a Pneumatic Artificial Muscle Using The Maxwell-Slip Model. Ieee-Asme Transactions On Mechatronics, 16(1), 177-186.

[14]. Liu, L., \& Yang, Y. (2015). Modeling and precision control of systems with hysteresis. ButterworthHeinemann 